\title{
The Developmental Changes of Number Processing and Calculation Abilities in Chinese Primary School Students
}

\author{
Shudong Zhang ${ }^{1}$, Fei Li ${ }^{1}$, Libo Zhao ${ }^{2 *}$, Lipei Xie ${ }^{3}$, Hui Zhao ${ }^{4,5^{*}}$ \\ ${ }^{1}$ Faculty of Education, Beijing Normal University, Beijing, CHINA \\ 2 Department of Psychology, BeiHang University, Beijing, CHINA \\ ${ }^{3}$ Beijing Jianxiang School, Beijing, CHINA \\ ${ }^{4}$ State Key Laboratory of Cognitive Neuroscience and Learning \& IDG/McGoven Institute for Brain Research, Beijing Normal \\ University, CHINA \\ ${ }^{5}$ Siegler Center for Innovations in Learning, Beijing Normal University, 100875, CHINA
}

Received 7 November 2017 • Revised 22 March 2018 - Accepted 27 March 2018

\begin{abstract}
Based on the 'triple-code' theory, the present study provided a comprehensive examination of the development of number processing and calculation abilities of Chinese primary school students. 310 children from grade 1 to grade 4 were assessed using the battery of number processing and calculation tests (NUCALC-R (Protocol)), covering tests of the Verbal, Visual Arabic and Analogue Magnitude Modules of the numerical abilities. The results showed that the three modules had different developmental trajectories from grade 1 to grade 4: the Verbal Module and Analog Module reached a plateau in grade 3, but the Visual Arabic Module improved gradually across the four grades. In addition, the subtests within each module also showed different developmental trajectories, demonstrating a rich profile of how the specific ways of representing and manipulating the numerals in a given module develop in the early school years.
\end{abstract}

Keywords: number processing and calculation, the triple-code model, development, NUCALC-R

\section{INTRODUCTION}

The importance of number processing and calculation abilities for an individual's success in the modern society makes it critical to understand how these abilities develop. The well-received triple-code model provides a comprehensive conceptual framework to examine the development of these numerical abilities, as it captures both the heterogeneity and the common structures among them. According to this model, the numerical abilities could be classified into three modules (i.e., the Analog Magnitude Module, the Verbal Module and the Visual Arabic Module), with each manipulating different forms of internal representations of numbers and being used for different numerical processing tasks (Dehaene, 1992). However, so far, little has been known about how these separate modules develop, as the existing studies have mainly focused on the development of one or just a few isolated abilities within a given module. The present investigation aims to delineate the developmental trajectories of these three modules of numerical cognition from grade 1 to grade 4 in the primary school. To serve this purpose, Neuropsychological Test Battery for Number Processing and Calculation in Children (NUCALC) was used (von Aster, 2000), which include different subtests tapping each of the three modules of the triple code model. Compared to the previous studies, this presents a more comprehensive picture of how the number processing and calculation abilities develop in the early years of schooling, both at the macro-level of modules and the micro-level of each single ability.

(c) 2018 by the authors; licensee Modestum Ltd., UK. This article is an open access article distributed under the terms and conditions of the Creative Commons Attribution License (http://creativecommons.org/licenses/by/4.0/). \zsd@bnu.edu.cn $\boldsymbol{\Delta}$ 49215075@qq.com $\boldsymbol{\nabla}$ libozhao@buaa.edu.cn (*Correspondence) 


\section{Contribution of this paper to the literature}

- In a single study we provide a comprehensive test of how three modules of number processing and calculation abilities develop in early years of schooling.

- This study also reveals how the constituent abilities within each module develop differently in a single study.

- It delineates a rich profile of the development of numerical processing abilities of Chinese primary school students.

\section{The Triple-Code Theory}

The overwhelming evidence of the heterogeneity of developmental dyscalculia indicates that the development of mathematical abilities is not a unidimensional process (e.g., Dehaene \& Cohen, 1997; Peake, Jimenez, \& Rodrigues, 2017). Therefore, we need a comprehensive conceptual framework to describe and explain the development of such multidimensional abilities.

The first such attempt was made by McCloskey and his colleagues (McCloskey, 1992; McCloskey, Caramazza, \& Basili, 1985). According to McClockey's model, numerical processing involved three separate systems that each corresponded to a specific cognitive function: the number comprehension system, calculation system, and number production system. According to this model, the comprehension system transforms numerical input into an abstract internal representation, i.e., the semantic code, which serves the input for the calculation system. In addition, the semantic codes generated by these two systems also provide input to the number production system that transforms the abstract codes into verbal (e.g., "seven") or Arabic number forms (e.g., "7"). Calculation involves operational words (more, less, times, divide) and operation symbols $(+,-, \times$ or $\div$ ), retrieval of them and arithmetic facts, and calculation procedures. Although the distinctions between these systems have been clearly supported by the neuropsychological evidences (e.g., McCloskey, 1992; McCloskey et al., 1985), the notion of exclusively abstract number representations was not supported by further analysis on the errors made by the braindamaged subjects (e.g., Campbell \& Clark, 1988), and also was inconsistent with the meta-analyses on $19 \mathrm{fMRI}$ studies on numerical cognition (Kaufmann, Wood, Rubinsten, \& Henik, 2011). Format-specific representations were thus incorporated in several alternative models (Campbell \& Clark, 1988; Dehaene, 1992; Dehaene, Piazza, Pinel, \& Cohen, 2003; Kaufmann et al., 2011; Noel \& Seron, 1993). However, the most influential one, the encoding-complex hypothesis goes to the other extreme by postulating a network of specific-format codes that were differentially recruited in different mathematical tasks for a given individual, depending upon an individual's idiosyncratic learning history, culture-specific strategies and other factors (Campbell \& Clark, 1988; Campbell \& Epp, 2004).

The 'triple-code' model proposed by Dehaene (1992) reconciled the two opposing perspectives on the number representations. This model proposes three distinct modules for number representation: the Analog Magnitude Module that is format-independent, and the Verbal Module and Visual Arabic Module that are format-dependent. The Analogical Magnitude Module represents numerical quantities analogically over a left-to-right oriented mental number line, and it is independent of language and available in preverbal infants and animals. According to this model, this module underlies automatic access to approximate quantities in the tasks of quantity comparison (e.g., "is five smaller than nine?") and approximation (e.g., estimate the number of apples in the box without counting). The Verbal Module represents numerical information in a verbal-presentation code (e.g., /thirteen/), which is created and manipulated by the language systems. This module is required for counting (e.g., count from 1 to 10), and tasks such as single-digit multiplication (e.g., " $5 \times 3=$ ?") and addition (e.g., "5+3=?") that involves direct retrieval of arithmetic facts from the long-term memory (e.g., addition and multiplication tables). The Visual Arabic Module, where strings of Arabic digits are encoded as Arabic codes (e.g., "13"), subserves parity judgments (e.g., "is 5 an odd number?") and multi-digit operations (e.g., " $56+120=$ ?"). It has been found that semantic knowledge of parity is accessed via a base-ten representation (i.e., the ten possible values of a number's rightmost digit) in Arabic form (Dehaene, Bossini, \& Giraux, 1993). The multi-digit operations involve the sequential combination of elementary arithmetical operations and thus are more complex. It has been documented that the understanding of the base-ten place-value structure in Arabic form is also critical (e.g., Nuerk, Weger, \& Willmes, 2001; for a review, see Klein, Bahnmueller et al., 2013). In addition, multi-digit calculations involve frequent translation between Arabic and verbal codes because direct retrieval of arithmetic facts is often needed, and also involve orienting of visual-spatial attention so as to mentally manipulate the spatial image of the operation in Arabic notation (Hubbard, Piazza, Pinel, \& Dehaene, 2005).

The triple-code model has been well supported by abundant of neural evidence (for a meta-analysis, see Kaufmann et al., 2011; for a review, see Siemann \& Petermann, 2017). The neuropsychological studies have revealed dissociations and double dissociations among these three systems. For example, patients with the left perisylvian damages were impaired in tasks requiring verbal representations of numbers, but could accomplish tasks involving 
quantity or Arabic representations (e.g., Dehaene \& Cohen, 1997; Lemer, Dehaene, \& Cohen, 2003). In contrast, the patients with parietal lesions were impaired in tasks involving quantity representations (e.g., Dehaene \& Cohen, 1997; Delazer \& Benke, 1997; Kaufmann et al., 2011). In addition, patients with pure alexic resulted from lesions in the inferior temporal gyrus failed to read aloud the visually presented digits and the operands, but could perform number comparison or old-even judgment, even with 2-digit numerals (Cohen \& Dehaene, 2000).

In line with the above neuropsychological evidence, the neuroimaging studies have also found that the Analog Magnitude, Verbal, and Visual Arabic modules are subserved by the bilateral inferior parietal, left perisylvian (e.g., angular gyrus and inferior frontal gyrus) and ventral occipitotemporal areas respectively (for a meta-analysis, see Kaufmann et al., 2011; for reviews, see Abboud, Maidenbaum, Dehaene \& Amedi, 2015; Siemann \& Petermann, 2017). For example, it has been revealed that Arabic digits and verbal numerals elicited similar activations in the inferior parietal lobe, suggesting a format-independent magnitude representation in this region (Holloway \& Anaari, 2008; Libertus, Woldorff, \& Brannon, 2007), but the Arabic digits elicited more activation in the inferior temporal gyrus than the verbal numerals (e.g., Shum et al., 2013). Further, a visual number form area (VNFA) that showed selectivity to Arabic digits was identified in the middle of the inferior temporal gyrus, which was close to the visual word form area (VWFA), but was connected to the intraparietal cortex that represented the quantities instead of the language areas (Abboud et al., 2015). In addition, tasks of approximate calculation and exact calculation led to greater activation in the parietal lobes and the inferior frontal lobe respectively (e.g., Dehaene et al., 1999, 2003). Using the exploratory group independent component analysis (ICA), another study processed the fMRI data from participants performing complex mental addition and subtraction of fractions and revealed separate task-related components in bilateral inferior parietal, left perisylvian and ventral occipitotemporal areas, also lending support to the triple-code model (Schmithorst \& Brown, 2004). Recently, the transcranial direct current stimulation (tDCS) was adopted and provided causal evidence for the links between these brain regions and these three codes (e.g., Artemenko, Moeller, Huber, \& Klein, 2015; Klein, Mann et al., 2013).

In sum, the dissociations of the three modules proposed by the triple-code model have been extensively reported by the neuroimaging and neuropsychological studies, making this theory be recognized as the most popular neuro-functional model for number processing and calculation (see Kaufmann et al., 2011). However, far more less has been known about how these separate modules develop, especially during the first few school years. In fact, in contrast to the above findings from the adult participants, studies on children failed to found dissociable neural circuits for the approximate and exact calculations (e.g., Kucian, von Aster, Loenneker, Dietrich, \& Martin, 2008; Molko et al., 2003), which suggests that these modules might undergo developmental changes over time.

\section{The Development of the Three Modules}

The previous studies on the development of the mathematical abilities have mainly been focusing on the developmental changes of a single or a few abilities in a single module, and thus could not inform how the modularized systems develop. For example, the studies on the development of the number comparison and estimation abilities have shown that the analog representation was in shape even with the newborn babies (Izard, Sann, Spelke, \& Streri, 2009; McCrink \& Wynn, 2004; Xu \& Spelke, 2000), and underwent a logarithmic-to-linear shift with age in representing numbers via a mental number line, indicating the refinement of education on this innate core system (Booth \& Siegler, 2006, 2008; Pinel \& Dehaene, 2013; Siegler \& Booth, 2004; von Aster \& Shalev, 2007). For another example, studies on how the mathematical abilities supported by the Verbal Module develop showed that counting was a preverbal ability and it matured before the school age (Wynn, 1992), and that calculation started with a strategy of finger counting at around four years old, moved up to adding without fingers with the minimum strategy at around 5 years old (Dehaene, 2011), and then to arithmetic facts retrieval, which became more automatized and in more complex forms during the preschool to the primary school years (Miller \& Paredes, 1990). For the third example, the development of multi-digit manipulation supported by the Visual Arabic Module received more attention in the recent years. It has been found that early understanding of place-value structure at grade 1 could predict the arithmetic performance at grade 3 (Moeller, Pixner, Zuber, Kaufmann, \& Nuerk, 2011). In another longitudinal study from grade 2 to grade 4 , the hundred-distance effect and the unithundred compatibility effect showed an increasing trend with grade level, suggesting more and more parallel processing of different positions (Mann, Moeller, Pixner, Kaufmann, \& Nuerk, 2012).

One might argue that these studies that focused on one or only a few abilities of numerical processing, when viewed collectively, could inform how the different modules and the constituent sub-abilities might differ in terms of the developmental trajectories. However, because these studies differed in numeral methodological aspects, it is simply impossible to make a fair comparison between them. As a result, these previous studies were not only unable to reveal the overall developmental patterns of the modules, but also were ineffective in revealing the variations of the sub-abilities within each module.

To serve the purpose of testing the three modules effectively in a single study, Neuropsychological Test Battery for Number Processing and Calculation in Children (NUCALC) was developed (von Aster, 2000). Functional 
analysis confirmed that sub-tests NUCALC could be organized into three clusters, fitting well with the three modules of Dehaene's triple code model (Santos et al., 2013; von Aster, 2000). There have been behavioral studies that used NUCALC to examine the age or grade effect on children across countries (Dellatolas, von Aster, Willadino-Braga, Meier, \& Deloche, 2000; Koumoula et al., 2004; Santos et al., 2013). These studies revealed an agerelated improvement in Score A, which consisted of six subtests that were associated with schooling achievement (dictation and reading of numbers, mental calculation addition part, problem solving, and oral and written comparison). However, by narrowing down the focus to these subtests, these studies did not inform how the three big modularized systems develop. In addition, by collapsing the subtest scores, these studies also did not reveal how the subtests that tapped different aspects within each module might differ in terms of the developmental patterns.

\section{The Present Study}

Therefore, based on the 'triple-code' theory, the present study aimed to adopt NUCALC-R (Protocol) to examine the developmental changes of three modules of numerical abilities in Chinese children from the grade 1 to grade 4 . In addition to comparing the overall developmental trajectories of the three modules across the grades, the present study also delineated the developmental variations among the subtests within each module. This will provide not only a better view of the modularized organization of the number processing and calculation in Chinese children, but also the variations of different aspects of representing and manipulating numerals in a particular code. Findings from this study will potentially fertilize more effective teaching practices in classrooms, which in turn will also help inform and refine the current models of the numerical cognition.

Based on the previous studies, we hypothesize that in general, children from the higher grades will perform better than those from the lower grades for all three modules. However, the grade effect might be lower for the Analogue Magnitude Module than the Verbal and Visual Arabic modules, as the former has been in position in the early infancy and thought of as innate abilities. In addition, the subtests within each module will demonstrate different developmental trajectories, determined by the difficulties of the specific ways of representing and manipulating the numerals in that code.

\section{METHOD}

\section{Participants}

310 primary school students from grade 1 to grade 4 were recruited from three schools in Beijing. The numbers in each grade was 40 (23 boys), 60 (37 boys), 81 (51 boys) and 129 ( 86 boys). None of these students had any physical deficits (e.g., hearing impairment) or mental retardation. The schools were evaluated to be good, average and below-average respectively based on the education level of school faculty, school facilities and the performance level of students. One class was randomly selected in each grade from grade 1 to grade 4 in all the selected schools except that for Grade 4 two classes were selected in the first school.

\section{Measurements}

The measurements were the Neuropsychological Test Battery for number Processing and Calculation in Children-revised (Protocol) (NUCALC-R (Protocol)) (von Aster \& Weinhold, 2002) that was translated and revised for use in China (Zhang \& Dong, 2006). Our previous study administered the NUCALC-R (Protocol) on Chinese primary school students and the confirmatory factor analysis revealed an acceptable fit with the 'triple-code' model (Zhang \& Dong, 2006). Specifically, the confirmatory factor analysis indicated that the Verbal Module includes dot enumeration (DE), verbal countdown (VC), mental calculation (MC), digit span (DS) and solving arithmetical problems (SAP); the Visual Arabic module includes transcription of dictated numbers (TDN), reading numbers (RN) and comparison of numbers as digit (transcribed) (CND); the Analog Magnitude Module comprises matching visual presentations of numerals to their corresponding positions on a vertical scale (MVPTCPVS), comparison of two numbers (verbally) (CTN), perceptual estimation of quantities (PEQ) and estimation of quantities in context (EQC). Below the subtests in each module are introduced.

\section{The verbal module}

Dot enumeration (DE). The child was asked to count aloud the dots displayed on two cards while pointing at each dot, and then write down the result. 
Verbal countdown (VC). The child was asked to count backwards from 23 to 1 . It requires producing the verbal sequence under the control of working memory and is a prerequisite for children to learn subtraction (Fuson, Richards, \& Briards, 1982).

Mental calculation $(\mathrm{MC})$. Addition (e.g., 5+8 =13) and subtraction (e.g., 32-17=15) were tested. Multiplication in the original version was excluded for lack of discriminative power on Chinese children. Probably due to extensive drilling of multiplication rules, Chinese children who have learned multiplication at school solved all the multiplication items correctly, whereas those haven't learnt solved none.

Digit span (DS). It included two parts, digit span forwards and digit span backwards. It was to measure the short-term storage and manipulation capacity of numbers.

Solving arithmetic problems (SAP). The child was asked to solve arithmetical problems of increasing difficulty. For example, "Peter has 12 marbles. He gives five to his friend Ann. How many marbles has he got left?"

\section{The visual Arabic module}

Reading numbers (RN). The child was asked to read aloud numbers written in Arabic numerals (e.g., 72). It required the child to transcode the numbers from the Arabic to the verbal form.

Transcription of dictated numbers (TDN). The child was asked to write down eight numbers (e.g., 14) in Arabic numerals that were presented orally by the experimenter.

Comparison of two numbers as digits (transcribed) (CTND). The numbers were presented in pair as Arabic numerals (e.g., 79 vs. 81). The child was asked to point out the larger one.

\section{The analog magnitude module}

Matching visual presentations of numerals to their corresponding positions on a vertical scale (MVPTCPVS). The child was asked to point to the small horizontal line that corresponds to a number presented as an Arabic numeral. The aim of this subtest was to examine the comprehension of the number as a quantity.

Comparison of two numbers (verbally) (CTNV). Pairs of numbers were presented orally. The child had to say which of the two numbers was larger.

Perceptual estimation of quantities (PEQ). The child was asked to orally estimate the quantity of objects in a picture that was presented for $5 \mathrm{~s}$ only. This subtest examined how numbers were associated with quantities in the child's internal representations.

Estimation of quantities in context (EQC). The child was asked to estimate quantities in specific sentential contexts (e.g., Two clouds in the sky) on a scale of three (a little, average, or a lot). This subtest examined the understanding of the semantic values of numbers in the specific contexts.

\section{Procedure}

A psychologist and trained schoolteachers administered the test battery. All of the 12 subtests were individually administered in quiet rooms. It took about 30 minutes for each child.

\section{Statistical Analysis}

The data were analyzed with SPSS 19. One-Way ANOVA was used to test the grade effects in the modules and subtests scores. Further, the post-hoc tests were used to reveal the grade differences in more details.

\section{RESULTS}

Each item in all the subtests scored between 0-2, and thus the average score for each subtest and each module was in the same range. All the following analysis was based on the averaged scores.

\section{The Development of the Verbal Module}

The Verbal Module includes the subtests of dot enumeration (DE), verbal countdown (VC), mental calculation (MC), digit span (DS) and solving arithmetical problems (SAP). The means and standard derivations of each of these subtests and the grand mean of the module for each grade are reported in Table 1 . The growth trajectories of the module mean scores were also displayed in Figure 1. 
Table 1. The Descriptive Analysis of the Verbal Module Subtests

\begin{tabular}{ccccc}
\hline Subtests & $\begin{array}{c}\text { Grade 1 } \\
\text { M (SD) }\end{array}$ & $\begin{array}{c}\text { Grade 2 } \\
\text { M (SD) }\end{array}$ & $\begin{array}{c}\text { Grade 3 } \\
\text { M (SD) }\end{array}$ & $\begin{array}{c}\text { Grade 4 } \\
\text { M (SD) }\end{array}$ \\
\hline DE & $1.54(.43)$ & $1.73(.40)$ & $1.79(.36)$ & $1.81(.34)$ \\
\hline VC & $1.09(.78)$ & $1.56(.55)$ & $1.65(.53)$ & $1.76(.42)$ \\
\hline MC & $0.87(.38)$ & $1.44(.35)$ & $1.62(.26)$ & $1.67(.25)$ \\
\hline DS & $1.38(.21)$ & $1.47(.23)$ & $1.59(.20)$ & $1.57(.21)$ \\
\hline SAP & $0.83(.57)$ & $1.20(.49)$ & $1.51(.44)$ & $1.60(.35)$ \\
\hline Verbal Total & $1.14(.32)$ & $1.48(.23)$ & $1.63(.21)$ & $1.68(.18)$ \\
\hline
\end{tabular}

Note. $\mathrm{DE}=$ dot enumeration; $\mathrm{VC}=$ verbal countdown; $\mathrm{MC}=$ mental calculation; $\mathrm{DS}=$ digit span; $\mathrm{SAP}=$ solving arithmetical problems.

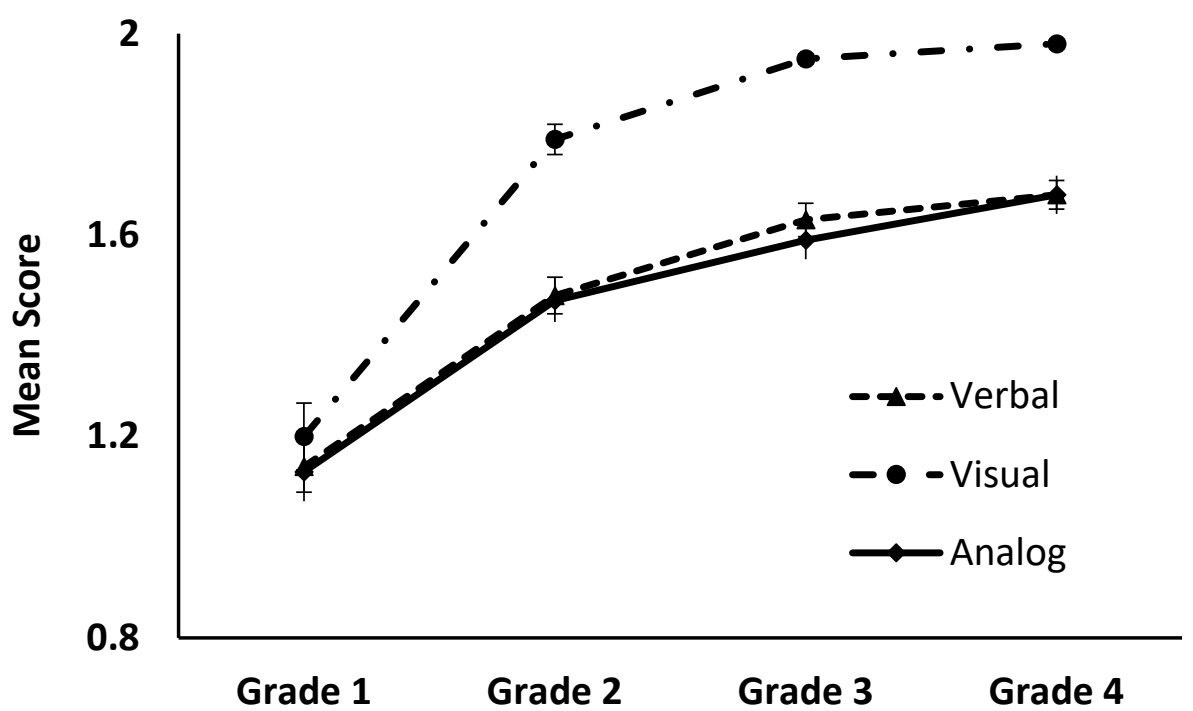

Figure 1. The growth curves of the three modules from grade 1 to grade 4

Table 2. One-Way ANOVA on the Grade Effect and Post-hoc Analysis for the Module and Subtest Scores of the Verbal Module

\begin{tabular}{cccc}
\hline Subtests & $\boldsymbol{d f}$ & Grade & Grade Comparisons \\
\hline $\mathrm{DE}$ & 3,306 & $6.00^{\star \star *}$ & Grade4, Grade3 \& Grade2 $>$ Grade1 \\
\hline $\mathrm{VC}$ & 3,306 & $16.55^{\star \star \star}$ & Grade4, Grade3 \& Grade2 $>$ Grade1 \\
\hline $\mathrm{MC}$ & 3,306 & $81.29^{\star \star \star}$ & Grade4 \& Grade3 $>$ Grade2 $>$ Grade1 \\
\hline $\mathrm{DS}$ & 3,306 & $12.55^{\star \star *}$ & Grade4 \& Grade3 $>$ Grade2 $>$ Grade1 \\
\hline SAP & 3,306 & $37.13^{\star \star *}$ & Grade4 \& Grade3 $>$ Grade2 $>$ Grade1 \\
\hline Verbal Total & 3,306 & $67.22^{\star * *}$ & Grade4 \& Grade3 $>$ Grade2 $>$ Grade1
\end{tabular}

Note. $\mathrm{DE}=$ dot enumeration; $\mathrm{VC}=$ verbal countdown; $\mathrm{MC}=$ mental calculation; $\mathrm{DS}=$ digit span; $\mathrm{SAP}=$ solving arithmetical problems. ${ }^{*} p<.05 .{ }^{* *} p<$ .01. ${ }^{* * *} p<.001$.

As the students from the four grades were independent groups, One-Way ANOVA was used to test the grade effects in the average module score and the subtest scores. The assumptions of One-Way ANOVA were also met (here and later for the rest modules). As Table 2 displays, this analysis revealed a significant grade effect in the average module score of the Verbal Module, $\mathrm{F}(3,306)=67.22, p<.001$. The grade effect for each subtest of this module was also significant (all ps<.001). The post-hoc tests further showed that grade 1 had significantly lower scores on all the subtests and module scores than the other grades (all $p s<.001$ ); grade 2 had significantly lower scores on all the subtest and module scores than grade 3 and grade 4 (all $p s<.001$ ), except that the scores of dot enumeration and verbal countdown were not significantly different from those of grade 3 (all $p s>.05$ ); grade 3 and grade 4 were equivalent across all the subtests and the module score (all $p s>.05$ ).

\section{The Development of the Visual Arabic module}

The Visual Arabic module includes the subtests of Transcription of dictated numbers (TDN), Reading numbers (RN), and Comparison of two numbers as digits (transcribed) (CTND). The means and standard deviations of the module score and the subtest scores for each grade were shown in Table 3 and Figure 1. 
Table 3. The Descriptive Analysis of the Visual Arabic Module Subtests

\begin{tabular}{ccccc}
\hline Subtests & $\begin{array}{c}\text { Grade 1 } \\
\text { M (SD) }\end{array}$ & $\begin{array}{c}\text { Grade 2 } \\
\text { M (SD) }\end{array}$ & $\begin{array}{l}\text { Grade 3 } \\
\text { M (SD) }\end{array}$ & $\begin{array}{c}\text { Grade 4 } \\
\text { M (SD) }\end{array}$ \\
\hline TDN & $0.9(.64)$ & $1.71(.37)$ & $1.96(.1)$ & $1.97(.08)$ \\
\hline RN & $1.11(.58)$ & $1.76(.25)$ & $1.96(.09)$ & $1.99(.05)$ \\
\hline CTND & $1.59(.35)$ & $1.9(.17)$ & $1.92(.14)$ & $1.98(.08)$ \\
\hline Visual Total & $1.2(.42)$ & $1.79(.19)$ & $1.95(.06)$ \\
\hline
\end{tabular}

Note. TDN=transcription of dictated numbers; RN=reading numbers; $C T N D=$ comparison of two numbers as digits (transcribed).

Table 4. One-Way ANOVA on the Grade Effect and Post-hoc Analysis for the Module and Subtest Scores of the Visual Arabic module

\begin{tabular}{cccc}
\hline Subtests & $\boldsymbol{d f}$ & Grade & Grade Comparisons \\
\hline TDN & 3,306 & $155.41^{\star \star \star}$ & Grade4 \& Grade3 $>$ Grade2 $>$ Grade1 \\
\hline RN & 3,306 & $147.55^{\star \star \star}$ & Grade4 \& Grade3 $>$ Grade2 $>$ Grade1 \\
\hline CTND & 3,306 & $53.73^{\star \star \star}$ & Grade4 $>$ Grade3 \& Grade2 $>$ Grade1 \\
\hline Visual Total & 3,306 & $214.48^{\star \star *}$ & Grade4 $>$ Grade3 $>$ Grade2 $>$ Grade1
\end{tabular}

Note. TDN=transcription of dictated numbers; RN=reading numbers; CTND= comparison of two numbers as digits (transcribed). ${ }^{\star} p<.05 .{ }^{* *} p<.01$. $* * * p<.001$

Table 5. The Descriptive Analysis of the Analogue Magnitude Module Subtests

\begin{tabular}{ccccc}
\hline Subtests & $\begin{array}{c}\text { Grade 1 } \\
\text { M (SD) }\end{array}$ & $\begin{array}{c}\text { Grade 2 } \\
\text { M (SD) }\end{array}$ & $\begin{array}{c}\text { Grade 3 } \\
\text { M (SD) }\end{array}$ & $\begin{array}{c}\text { Grade 4 } \\
\text { M (SD) }\end{array}$ \\
\hline MVPNTCPVS & $1.16(.68)$ & $1.54(.46)$ & $1.65(.42)$ & $1.71(.41)$ \\
\hline CTNV & $1.22(.59)$ & $1.70(.25)$ & $1.85(.22)$ & $1.84(.23)$ \\
\hline PEQ & $1.22(.62)$ & $1.50(.57)$ & $1.56(.54)$ & $1.72(.45)$ \\
\hline EQC & $0.94(.4)$ & $1.13(.43)$ & $1.28(.45)$ & $1.44(.41)$ \\
\hline Analogue Total & $1.13(.37)$ & $1.47(.27)$ & $1.59(.24)$ & $1.68(.24)$ \\
\hline
\end{tabular}

Note. MVPNTCPVS=matching visual presentations of numerals to their corresponding positions on a vertical scale; CTN=comparison of two numbers (verbally); $\mathrm{PEQ}=$ perceptual estimation of quantities; $\mathrm{EQC}=$ estimation of quantities in context

As Table 4 displays, one-way ANOVA revealed a significant grade effect in the average module score of the Visual Arabic module, F $(3,306)=214.48, p<.001$. The grade effect for each subtest of this module was also significant (all ps <.001). The post-hoc test showed that the module score increased significantly as the grade increased (all ps <.05). The same was true for the comparison of two numbers as digits (transcribed) (CTND), but for transcription of dictated numbers and reading numbers (TDN), there was a significant increase except from grade 3 to grade 4 .

\section{The Development of the Analog Magnitude Module}

The Analog Magnitude Module includes the subtests of matching visual presentations of numerals to their corresponding positions on a vertical scale (MVPNTCPVS), comparison of two numbers verbally (CTN), perceptual estimation of quantities and estimation of quantities in context (EQC). The means and standard deviations of the module score and the subtest scores for each grade were shown in Table 5 and Figure $\mathbf{1 .}$

As Table 6 shows, One-Way ANOVA revealed a significant grade effect for the overall score of the Analog Magnitude Module, F $(3,306)=44.5, p<.001$, and for all of its subtests (all ps<.001). The post-hoc tests showed that the module score reached asymptote at grade 3 . The same was true for Comparison of two numbers as digits (transcribed). The subtest of perceptual estimation of quantities seems to be the easiest, as it stopped growing at grade 2, and Estimation of quantities in contest seems to be the hardest, as it had a significant increase from every grade to its next $(p<.001)$. The subtest of matching visual presentations of numerals to their corresponding positions on a vertical scale increased from grade 1 to grade 2 , ceased to grow from grade 2 to grade 3 , but continued to increase from grade 3 to grade 4 . 
Table 6. One-Way ANOVA on the Grade Effect and Post-hoc Analysis for the Module and Subtest Scores of the Analogue Magnitude Module

\begin{tabular}{|c|c|c|c|}
\hline Subtests & $d f$ & Grade & Grade Comparisons \\
\hline MVPNTCPVS & 3,306 & $14.87^{\star \star *}$ & Grade4, Grade3 \& Grade2 > Grade1 \\
\hline CTNV & 3,306 & $47.75^{\star * *}$ & Grade4 \& Grade3 > Grade2 > Grade1 \\
\hline PEQ & 3,306 & $9.86^{\star \star \star}$ & Grade4 \& Grade3 \& Grade2 > Grade1 \\
\hline EQC & 3,306 & $17.09^{\star * *}$ & Grade4 $>$ Grade3 > Grade2 > Grade1 \\
\hline Analogue Total & 3,306 & $44.5^{\star \star \star}$ & Grade4 \& Grade3 > Grade2 > Grade1 \\
\hline
\end{tabular}

Note. MVPNTCPVS=matching visual presentations of numerals to their corresponding positions on a vertical scale; $C T N=$ comparison of two

numbers (verbally); $\mathrm{PEQ}=$ perceptual estimation of quantities; $\mathrm{EQC}=$ estimation of quantities in context. ${ }^{*} p<.05 .{ }^{* *} p<.01 .{ }^{* * *} p<.001$.

\section{DISCUSSION}

The present study for the first time examined the developmental changes for the Visual, Verbal and Analog Magnitude Module of numerical processing and calculation from grade 1 to grade 4 in the primary school. The results showed that the three modules had different patterns of changes across the four years: whereas the performance of the Visual Arabic module increased from grade 1 to grade 4, both the verbal and Analog Magnitude Module reached a plateau at grade 3. In addition, the subtests within each module also showed different developmental trajectories across the four grades, revealing a rich profile of how the specific ways of representing and manipulating the numerals in a given code develop in the early school years.

The Verbal Module score was the lowest in grade 1, improved significantly from grade 1 to grade 2 , and then reached a plateau at grade 3 . The growth from grade 1 to grade 2 might be driven by multiple factors, such as more arithmetic facts being acquired during the first year of formal education, accumulation of experiences, and the development of processing speed (e.g., Fry \& Hale, 1996). The plateau observed at grade 3 was similar to that observed in Greek children with the same protocol of NUCALC (Koumoula et al., 2004). A possible explanation for this phenomenon was that the tests might be too easy for the higher graders, as they were designated to diagnose children with the developmental dyscalculia (von Aster, 2000). Interestingly, here we found that the plateau occurred one year earlier than that in Greek children. This fits with the extensive cross-cultural evidence that Chinese children enjoy an advantage in mathematics over the Western peers, presumably because the verbal forms of digits in Chinese are shorter than those in the alphabetical languages and presents a less load for the verbal shortterm memory (Dehaene, 2011). As for the individual subtests of the Verbal Module, all improved significantly from grade 1 to grade 2, but their developmental trajectories bifurcated thereafter. Dot enumeration and verbal countdown did not improve further after grade 2 , which was consistent with previous evidence that counting was a precocious competence and served a foundation for the development of exact calculation (Wynn, 1992). Mental calculation, solving arithmetical problems and digit span showed steady improvement from grade 1 to grade 3 , and emergence of stagnation in grade 3 and grade 4 .

The Visual Arabic Module presented a gradual and continuous improvement from grade to grade. Although the amount of growth decreased across the grades and was in fact was small from grade 3 to grade 4 , all the changes were statistically significant. There might be two main drives underneath this gradual improvement of this module across grades. The first drive might be children's continuous acquisition of the Arabic notation system (e.g., the base-ten place-value structure) and written calculation procedures with increased difficulty in the school environment (Knops, Thirion, Hubbard, Michel, \& Dehaene, 2009). The second drive could be the increasingly developed visual and spatial attention (e.g., Shimi, Nobre, \& Astle, 2014). It has been revealed that mathematical processing, especially multi-digit manipulation requires the orientation of the visual-spatial attention (Hubbard et al., 2005; Maruyama et al., 2012) and that for children with Attention-Deficit Hyperactivity Disorder (ADHD), decrements in sustained attention could predict mathematical performance (Fosco \& Hawk, 2017). Future studies should further explore the causal role of visual-spatial attention in the development of the Visual Arabic Module by using a longitudinal design or a training paradigm.

The Analog Magnitude Module overall showed a similar developmental trajectory with that of the Verbal Module, presenting a continuous growth till a plateau was reached at grade 3 . This pattern suggests that the Analog module might have reached a stable state at grade 3 , corroborating with one recent imaging finding that the thirdand sixth-graders had no significant differences in their brain activations when performing approximate calculation and magnitude comparison (Kucian, von Aster, Loenneker, Dietrich, \& Martin, 2008). The individual subtests of the Analog Magnitude Module all improved significantly from grade 1 to grade 2, but their developmental trajectories thereafter were different from test to test. Perceptual estimation of quantities and matching visual presentations of numerals to their corresponding positions on a vertical scale stopped to grow at grade 2 . This suggests that although the ability of perceiving quantity has been in shape before schooling or even at birth as the previous studies suggested (Cantlon, Brannon, Carter, \& Marrero, 2006; Wynn, 1992; Xu \& Spelke, 2000), it could 
be refined by education. The comparison of two digits (verbally) did not stop until grade 3, whereas estimation of quantities in context showed a continuous growth from grade 1 to grade 4 . Such developmental changes can be interpreted as a refinement of the quantitative representation after exposures to numerical symbols, presumably in the left IPS of the brain (Pinel \& Dehaene, 2013). This paralleled with the view of the four-step-developmental model of numerical cognition, which suggested the elaboration of the Analog Magnitude Module after symbolization of numbers during the school age (von Aster \& Shalev, 2007).

\section{LIMITATIONS}

The present study had at least two limitations. First, it used a cross-sectional method to examine the developmental changes and thus the cohort effect might be an issue. Second, although our sample included the atrisk students, due to the small sample size we didn't examine whether they followed a different developmental pattern from the average ones for these three modules and the subtests. To overcome these limitations, we are currently planning a longitudinal study to trace the developmental patterns of number processing and calculation of both the typically developed and the at-risk students, and to delineate when, where and how much the latter deviates from the former.

\section{CONCLUSION}

In sum, the results of the present study indicated that the developmental changes of different modules of number processing and calculation were not of a uniform pattern. However, two commonalities did emerge. First, all three modules improved with grade, which was presumably driven jointly by maturation and experience (e.g., Izard, Sann, Spelke, \& Streri, 2009). Second, the developmental trajectories of the subtests within each module were not uniform: some easier subtests matured early on in grade 2, but the more difficult ones did not reach plateau until grade 4 or even later. These developmental changes help draw a better and more comprehensive picture of the mathematical abilities of Chinese children in each grade. This will help maths teachers to more accurately estimate the developmental levels of different aspects of mathematical abilities and to identify the zone of proximal development(ZPD) (Vygotsky, 1986) of each, so as to adjust their paces of strategies of teaching to promote students' learning (Holton \& Clarke, 2006).

\section{ACKNOWLEDGEMENTS}

The authors disclosed receipt of the following financial support for the research, authorship, and/or publication of this article: Beijing Education and Science Project 2013 (ABA13014); The key project of Ministry of Education (DBA130218); An opening project of National Key Laboratory of Cognitive Neuroscience and Learning, Beijing Normal University, Beijing (KF2011YB01); a National Natural Science Foundation of China (31300854); 2018 Comprehensive Discipline Construction Fund of Faculty of Education, Beijing Normal University. The authors would also like to thank Xia, Xuenan for help with literature survey and editing.

\section{REFERENCES}

Abbound, S., Maidenbaum, S., Dehaene, S., \& Amedi, A. (2015). A number-form area in the blind. Nature Communication, 6, 6026. https:// doi.org/10.1038/ncomms7026

Artemenko, C., Moeller, K., Huber, S., \& Klein, E. (2015). Differential influences of unilateral tDCS over the intraparietal cortex on numerical cognition. Frontiers in Human Neuroscience, 9, 1-8. https:/ / doi.org/10.3389/fnhum.2015.00110

Booth, J. L., \& Siegler, R. S. (2006). Developmental and individual differences in pure numerical estimation. Developmental Psychology, 42(1), 189-201. https:/ / doi.org/10.1037/0012-1649.41.6.189

Booth, J. L., \& Siegler, R. S. (2008). Numerical magnitude representations influence arithmetic learning. Child Development, 79(4), 1016-1031. https:/ / doi.org/10.1111/j.1467-8624.2008.01173.x

Campbell, J., \& Clark, J. (1988). An encoding-complex view of cognitive number processing: Comment on McCloskey, Sokol, and Goodman (1986). Journal of Experimental Psychology: General, 117(2), $204-214$. https:/ / doi.org/10.1037/0096-3445.117.2.204

Campbell, J., \& Epp, L.J. (2004). An Encoding-Complex Approach to Numerical Cognition in Chinese-English Bilinguals. Canadian Journal of Experimental Psychology, 58:4, 229-244. https:/ / doi.org/10.1037/h0087447

Cantlon, J. F., Brannon, E. M., Carter, E. J., \& Pelphrey, K. A. (2006). Functional imaging of numerical processing in adults and 4-y-old children. PLoS Biology, 4(5), 844-854. https:/ / doi.org/10.1371/journal.pbio.0040125 
Cohen, L., \& Dehaene, S. (2000). Calculating without reading: unsuspected residual abilities in pure alexia. Cogntive Neuropsycholgy 17(6), 563-583. https:/ / doi.org/10.1080/02643290050110656

Dehaene, S. (1992). Varieties of numberical abilities. Cognition, 44, 1-42. https://doi.org/10.1016/00100277(92)90049-N

Dehaene, S. (2011). The number sense: how the mind creates mathematics: Oxford University Press.

Dehaene, S., \& Cohen, L. (1997). Cerebral pathways for calculation: Double dissociation between rote verbal and quantitative knowledge of arithmetic. Cortex; A Journal Devoted to the Study of the Nervous System and Behavior, 33(2), 219-250. https:// doi.org/10.1016/S0010-9452(08)70002-9

Dehaene, S., Bossini, S., \& Giraux, P. (1993). The Mental Representation of Parity and Number Magnitude. Journal of Experimental Psychology: General, 122(3), 371-396. https:/ / doi.org/10.1037/0096-3445.122.3.371

Dehaene, S., Piazza, M., Pinel, P., \& Cohen, L. (2003). Three parietal circuits for number processing. Cognitive Neuropsychology, 20(3), 487-506. https:/ / doi.org/10.1080/02643290244000239

Dehaene, S., Spelke, E., Pinel, P., Stanescu, R., \& Tsivkin, S. (1999). Sources of mathematical thinking: behavioral and brain-imaging evidence. Science, 284(5416), 970-974. https://doi.org/10.1111/j.14679280.1996.tb00366.x

Delazer, M., \& Benke, T. (1997). Arithmetic facts without meaning. Cortex, 33(4), $697-710$. https:/ / doi.org/10.1016/S0010-9452(08)70727-5

Dellatolas, G., von Aster, M., Willadino-Braga, L., Meier, M., \& Deloche, G. (2000). Number processing and mental calculation in school children aged 7 to 10 years: a transcultural comparison. European Child and Adolescent Psychiatry, 9 Suppl 2, II102-110. https:/ / doi.org/10.1007/s007870070003

Fosco, W. D., \& Hawk, L. W. (2017). Relating lab to life: Decrements in attention over time predict math productivity among children with ADHD. Child Neuropsychology, 23(2), 148-158. https:/ / doi.org/10.1080/09297049.2015.1089982

Fry, A. E., \& Hale, S. (1996). Processing speed, working memory, and fluid intelligence: Evidence for a developmental cascade. Psychological Science, 7, 237-241. https://doi.org/10.1111/j.14679280.1996.tb00366.x

Fuson, K. C., Richards, J., \& Briards, D. J. (1982). The Acquisition of the Number Word Sequence. In C. J. Brainerd (Hrsg.), Children's Logical and Mathematical Cognition. Progress in Cognitive Development Research (S. 33-92). New York: Springer.

Holloway, I., \& Ansari, D. (2008). Mapping numerical magnitudes onto symbols: The numerical distance effect and individual differences in children's mathematics achievement. Journal of Experimental Child Psychology, 103, 17-29. https:// doi.org/10.1016/j.jecp.2008.04.001

Holton, D., \& Clarke, D. (2006). Scaffolding and metacognition. International Journal of Mathematical Education in Science E Technology, 37(2), 127-143. https:/ / doi.org/10.1080/00207390500285818

Hubbard, E. M., Piazza, M., Pinel, P., \& Dehaene, S. (2005). Interactions between number and space in parietal cortex. Nature Reviews Neuroscience, 6(6), 435-448. https:/ / doi.org/10.1038/nrn1684

Izard, V., Sann, C., Spelke, E. S., \& Streri, A. (2009). Newborn infants perceive abstract numbers. Proceedings of the National Academy of Sciences of the United States of America, 106(25), 10382-10385. https:// doi.org/10.1073/pnas.0812142106

Kaufmann, L., Wood, G., Rubinsten, O., \& Henik, A. (2011). Meta-analyses of developmental fMRI studies investigating typical and atypical trajectories of number processing and calculation. Developmental Neuropsychology, 36(6), 763-787. https://doi.org/10.1080/87565641.2010.549884

Klein, E., Bahnmueller, J., Mann, A., Pixner, S., Kaufmann, L., Nuerk, H. C., \& Moeller, K. (2013). Language influences on numerical development-inversion effects on multi-digit number processing. Frontiers in Psychology, 4(8), 1-6. https:/ / doi.org/10.3389/ fpsyg.2013.00480

Klein, E., Mann, A., Huber, S., Bloechle, J., Willmes, K., Karim, A. A., .. Moeller, K. (2013). Bilateral Bi-Cephalic Tdcs with Two Active Electrodes of the Same Polarity Modulates Bilateral Cognitive Processes Differentially. Plos One, 8 (8), e71607. https:// doi.org/10.1371/journal.pone.0071607

Knops, A., Thirion, B., Hubbard, E. M., Michel, V., \& Dehaene, S. (2009). Recruitment of an area involved in eye movements during mental arithmetic. Science, 324(5934), 1583-1585. https:/ / doi.org/10.1126/science.1171599

Koumoula, A., Tsironi, V., Stamouli, V., Bardani, I., Siapati, S., Annika, G., ... von Aster, M. (2004). An epidemiological study of number processing and mental calculation in Greek schoolchildren. Journal of Learning Disabilities, 37(5), 377-388. https:/ / doi.org/10.1177/00222194040370050201 
Kucian, K., von Aster, M., Loenneker, T., Dietrich, T., \& Martin, E. (2008). Development of neural networks for exact and approximate calculation: a FMRI study. Developmental Neuropsychology, 33(4), 447-473. https:// doi.org/10.1080/87565640802101474

Lemer, C., Dehaene, S., Spelke, E., \& Cohen, L. (2003). Approximate quantities and exact number words: Dissociable systems. Neuropsychologia, 41, 1942-1958. https:/ / doi.org/10.1016/S0028-3932(03)00123-4

Libertus, M., Woldorff, M. G, \& Brannon, E. M. (2007). Electrophysiological evidence for notation independence in numerical processing. Behavioral and Brain Functions, 3, Article 1. https:/ / doi.org/10.1186/1744-9081-3-1

Mann, A., Moeller, K., Pixner, S., Kaufmann, L., \& Nuerk, H. C. (2012). On the development of Arabic three-digit number processing in primary school children. Journal of Experimental Child Psychology, 113(4), 594-601. https://doi.org/10.1016/j.jecp.2012.08.002

Maruyama, M., Pallier, C., Jobert, A., Sigman, M., \& Dehaene, S. (2012). The cortical representation of simple mathematical expressions. NeuroImage, 61(4), 1444-1460. https:/ / doi.org/10.1016/j.neuroimage.2012.04.020

McCloskey, M. (1992). Cognitive mechanisms in numerical processing: evidence from acquired dyscalculia. Cognition, 44(1-2), 107-157. https:/ / doi.org/10.1016/0010-0277(92)90052-J

McCloskey, M., Caramazza, A., \& Basili, A. (1985). Cognitive mechanisms in number processing and calculation: evidence from dyscalculia. Brain and Cog., 4(2), 171-196. https:/ / doi.org/10.1016/0278-2626(85)90069-7

McCrink, K., \& Wynn, K. (2004). Large-number addition and subtraction by 9-month-old infants. Psychological Science, 15(11), 776-781. https:/ / doi.org/10.1111/j.0956-7976.2004.00755.x

Miller, K. F., \& Paredes, D. R. (1990). Starting to add worse: effects of learning to multiply on children's addition. Cognition, 37(3), 213-242. https:/ / doi.org/10.1016/0010-0277(90)90046-M

Moeller, K., Pixner, S., Zuber, J., Kaufmann, L., \& Nuerk, H.-C. (2011). Early place-value understanding as a precursor for later arithmetic performance. A longitudinal study on numerical development. Research in Developmental Disabilities, 32, 1837-1851. https:// doi.org/10.1016/j.ridd.2011.03.012

Molko, N., Cachia, A., Riviere, D., Mangin, J. F., Bruandet, M., Le Bihan, D., ... Dehaene, S. (2003). Functional and structural alterations of the intraparietal sulcus in a developmental dyscalculia of genetic origin. Neuron, 40(4), 847-858. https:/ / doi.org/10.1016/S0896-6273(03)00670-6

Noel, M.-P., \& Seron, X. (1993). Arabic number reading deficit: A single case study or when 236 is read (2306) and judged superior to $1258 . \quad$ Cognitive Neuropsychology, 10(4), 317-339. https:/ / doi.org/10.1080/02643299308253467

Nuerk, H.-C., Weger, U., \& Willmes, K. (2001). Decade breaks in the mental number line? Putting the tens and units back in different bins. Cognition, 82, B25-B33. https:/ / doi.org/10.1016/S0010-0277(01)00142-1

Peake, C., Jimenez, J. E., \& Rodrigues, C. (2017). Data-driven heterogeneity in mathematical learning disabilities based on the triple code model. Research in Developmental Disabilities, 71, 130-142. http://doi.org/10.1016/j.ridd.2017.10.005

Pinel, P., \& Dehaene, S. (2013). Genetic and environmental contributions to brain activation during calculation. NeuroImage, 81, 306-316. https:/ / doi.org/10.1016/j.neuroimage.2013.04.118

Santos, F. H. D., Da Silva, P. A., Ribeiro, F. S., Dias, A. L. R. P., Frigério, M. C., Dellatolas, G., \& von Aster, M. (2013). Number processing and calculation in Brazilian children aged 7-12 years. The Spanish Journal of Psychology, 15(2), 513-525. https:// doi.org/10.5209/rev_SJOP.2012.v15.n2.38862

Schmithorst, V. J., \& Brown, R. D. (2004). Empirical validation of the triple-code model of numerical processing for complex math operations using functional MRI and group Independent Component Analysis of the mental addition and subtraction of fractions. NeuroImage, 22(3), 1414-1420. https://doi.org/10.1016/j.neuroimage.2004.03.021

Shimi, A., Nobre, A. C., Astle, D., \& Scerif, G. (2014). Orienting Attention within Visual Short-Term Memory: Development and Mechanisms. Child development, 85(2), 578-592. https:/ / doi.org/10.1111/cdev.12150

Shum, J., Hermes, D., Foster, B. L., Dastjerdi, M., Rangarajan, V., Winawer, J., ... Parvizi, J. (2013). A brain area for visual numerals. J. Neurosci. 33, 6709-6715. https:// doi.org/10.1523/JNEUROSCI.4558-12.2013

Siegler, R. S., \& Booth, J. L. (2004). Development of numerical estimation in young children. Child Development, 75(2), 428-444. https:/ / doi.org/10.1111/j.1467-8624.2004.00684.x

Siemann, J., \& Petermann, F. (2017). Evaluation of the Triple Code Model of numerical processing - Reviewing past neuroimaging and clinical findings. Research in Developmental Disabilities, 72, 106-117. https://doi.org/10.1016/j.ridd.2017.11.001 
von Aster, M. (2000). Developmental cognitive neuropsychology of number processing and calculation: varieties of developmental dyscalculia. European Child $\mathcal{E}$ Adolescent Psychiatry, 9 Suppl 2, II41-57. https:/ / doi.org/10.1007/s007870070008

von Aster, M., \& Shalev, R. (2007). Number development and developmental dyscalculia. Developmental Medicine and Child Neurology, 49(11), 868-873. https:/ / doi.org/10.1111/j.1469-8749.2007.00868.x

von Aster, M., \& Weinhold, M. (2002). The neuropsychological test battery for number processing and calculation in children (NUCALC-R) PROTOCOL. Männedorf.

Vygotsky, L. S. (1986). In Thought and Language (Kozulin, A., ed.), MIT Press.

Wynn, K. (1992). Children's acquisition of the number words and the counting system. Cognitive Psychology, 24(2), 220-251. https://doi.org/10.1016/0010-0285(92)90008-p

Xu, F., \& Spelke, E. S. (2000). Large number discrimination in 6-month-old infants. Cognition, 74(1), B1-B11. https:/ / doi.org/10.1016/S0010-0277(99)00066-9

Zhang, S., \& Dong, Q. (2006). Revision of test of number processing and calculation and study on its reliability and validity. Chinese Journal of Special Education, 71(5), 62-66.

\section{http://www.ejmste.com}

